\title{
The Role of the Ventral Medial Prefrontal Cortex in Social Decision Making
}

\author{
Wouter van den Bos ${ }^{1,2}$ and Berna Güroğlu ${ }^{1,2}$ \\ ${ }^{1}$ Institute of Psychology, Leiden University, 2333 AK Leiden, The Netherlands, and ${ }^{2}$ Leiden Institute for Brain and Cognition, 2300 RC Leiden, \\ The Netherlands \\ Review of Krajbich et al. (http://www.jneurosci.org/cgi/content/full/29/7/2188)
}

Convergent evidence has revealed that the ventromedial prefrontal cortex (VMPFC) is important for social decision making; however, its exact function is still under debate. Studying behavioral performance of patients with VMPFC lesions is one important way of learning about the role of VMPFC in social processing. Lesions to the VMPFC seriously disrupt social life by affecting decision-making abilities and emotion processing. The deficits in decision making were first demonstrated by a series of studies of patients with VMPFC lesions which have shown that they are particularly impaired in making valuebased decisions (Damasio, 1994). This set of studies has shown that these patients are insensitive to future consequences, positive and negative, and are primarily guided by immediate gains (Bechara et al., 2000). Recent research has demonstrated that VMPFC patients also differ from control subjects in their behavior on a range of social tasks, including moral judgment (Koenigs et al., 2007) and economic games (Koenigs and Tranel, 2007); they make more irrational economic decisions and show a lack of emotional reactions. These findings extend previous knowledge on the role of VMPFC in deci-

\footnotetext{
Received April 16, 2009; revised May 13, 2009; accepted May 13, 2009. We thank Eveline Crone and Eric van Dijk for their helpful comments. Correspondence should be addressed to Wouter van den Bos, Institute of Psychology, Leiden University, Wassenaarseweg 52, 2333 AK Leiden, The Netherlands. E-mail:WBos@fsw.leidenuniv.nl.

DOI:10.1523/JNEUROSCI.1821-09.2009

Copyright $\odot$ 2009 Society for Neuroscience $\quad$ 0270-6474/09/297631-02\$15.00/0
}

sion making and suggest that VMPFC deficits are related to impaired abilities to either generate (Koenigs et al., 2007) or to regulate (Koenigs and Tranel, 2007) emotions. However, others suggest that the deficits in social decision making might be caused by an impaired "mentalizing" ability (Shamay-Tsoory et al., 2007). In short, it is still not clear what the underlying mechanisms of their deficient social behavior are.

In their recent article in The Journal of Neuroscience, Krajbich et al. (2009) were one of the first to set out to test the role of two specific emotions, envy and guilt, in social deficits of VMPFC patients. In this study, the authors employ a model-based approach using three different economic games, the dictator game, ultimatum game, and trust game. In the dictator game each participant was asked to divide 50 points between herself and an anonymous stranger. In the ultimatum game, participants were again asked to divide 50 points; however, this time the recipient of the offer could either accept or reject it (in the latter case neither player gets anything). All participants played the ultimatum game twice, once as the proposer and once as the responder. Finally, participants played several rounds of the trust game, in which the first player (investor) chooses to either trust or to not trust the (anonymous) other player (trustee) to divide the stake between the two players. If the investor chooses not to trust, players share the original stake equally and the game ends. If the investor chooses to trust, the stake is increased and subsequently the trustee can either decide to repay (sharing the money equally) or to betray trust (taking all the money for herself). The temptation to betray trust was manipulated across the different versions of the trust game [Krajbich et al. (2009), their supplemental Fig. 1]. All participants played each version of the trust game both as investor and trustee. They were told that there was a transformation from points to money at the end of the session. All games were played by three sets of participants: VMPFC patients, brain damage controls, and normal controls.

The combination of the results from the different economic games and a model of choice behavior allowed the authors to infer the social preference parameters of each individual. The model is able to put a weight (parameter value) on the difference in earnings when others earn more (envy) or less (guilt) than oneself. The higher the weight on guilt, the less the participant prefers to earn more than the other, as measured by the amount offered in the dictator game and the ultimatum game and the trustee decisions to repay in the trust game. The higher the weight on envy, the less the participants prefers to earn less than the other, as measured by the investor decision to trust in the trust game. In addition to these preferences, the model also incorporates estimates of par- 
ticipants' expected guilt and envy from the other player.

The VMPFC patients differed from the brain damage controls and normal controls in several ways. First, they gave significantly less in the dictator game; only 4.7 out of 50 , compared with 12 and 18 for the brain damage controls and normal controls, respectively. These findings suggest less feelings of guilt associated with lower levels of prosocial behavior. Second, in the ultimatum game, VMPFC patients did not differ from the two control groups in offers made as proposers and minimal acceptable offers they indicated as responders. However, VMPFC patients deviated from the controls by not offering more than they demanded themselves in the ultimatum game, whereas controls offered more than they demanded. This result confirmed the authors' expectation of less guilt felt by VMPFC patients since offering more than what one believes to be a minimal acceptable offer suggests feelings of guilt. Third, in the trust game, the VMPFC group showed less trust and less trustworthiness (rate of repayment) than the normal controls, but did not differ on trustworthiness compared with the brain damage controls [Krajbich et al. (2009), their Table 1], confirming expectations of guilt and envy by VMPFC patients, but also suggesting that other brain regions may also play a role here.

Parametric analyses further revealed that the VMPFC group differed on the guilt parameter from the two control groups, but did not differ on the envy parameter. This is in-line with previous theories on VMPFC damage that indicate that these patients lack guilt but do not differ on other types of emotions (Koenigs et al., 2007). Although the VMPFC patients differed in their experienced guilt, they did not differ on the expected guilt and expected envy parameters, indicating that lower levels of guilt experienced by VMPFC patients are not caused by differences in their "mentalizing" abilities. Together, the findings of the Krajbich et al. (2009) study indicate that the behavior of VMPFC lesion patients is instead resulting from the inability to generate a specific type of emotion (guilt). Although this finding gives us more insight on the role of VMPFC in social cognition, the precise mechanism behind differences in the social preferences of VMPFC patients remains unknown; it is still unclear why damage to the VMPFC would impair some emotions and not others.

Given that VMPFC patients are also impaired in making decisions that involve future consequences (Damasio, 1994), the examination of the temporal aspects of social decision making might facilitate our understanding of the role of VMPFC in social cognition. A recent study (Moretti et al., 2009) tests the role of temporal aspects of incentives in accepting unfair offers by offering concrete, immediate or abstract, future rewards in the ultimatum game. They find that VMPFC patients reject unfair offers more often only when rewards are abstract and delayed and not when immediate and concrete rewards (dollar bills) are presented. This finding suggests that damage to the VMPFC reduces the motivation to obtain future rewards and results in an inability to adapt behavior according to long-term consequences. In the study by Koenigs and Tranel (2007), as well as that by Krajbich et al. (2009), rewards were both abstract and delayed; participants "earned" points in the game and did not know how they would be converted to real money.

The diminished sensitivity to abstract decision outcomes and delayed consequences in VMPFC patients might warrant reinterpretation of the Krajbich et al. (2009) study. Feelings of guilt can be seen as expected future punishments related to outcomes of one's behavior. In the social decision-making paradigms, feelings of guilt are expected negative feelings resulting from future consequence of one's decisions, whereas feelings of envy are current feelings resulting from one's status compared with that of the other. If VMPFC patients have trouble representing future and abstract consequences of their decisions, they might have problems incorporating expected guilt into their decision-making process. Similarly, they might have trouble representing positive feelings or concrete earnings as expected future consequences (rewards) of cooperative behavior. Thus, it would be promising for future research to focus on investigating temporal preferences of the VMPFC patient group by studying deci- sion making with delayed rewards as well as delayed punishments.

Furthermore, future rewards could be made more salient in a social setting by using repeated games. Not only are repeated games ecologically more valid, most people are more cooperative in repeated games because they know that this might increase their pay off in long term. For instance, if it is the lack of guilt that is driving the "selfish" behavior in the trust game, this should be abolished when playing a repeated game because one would expect strategic future oriented behavior to take over. If VMPFC patients indeed have problems with representing future rewards, one would expect them to keep on betraying in a repeated trust game as well.

It is possible that VMPFC patients have difficulty with both the generation of certain emotions and the representation of future outcomes. A model that incorporates both factors could provide us with more insight in what the relative contribution of these factors is in a given experimental context. The study by Krajbich et al. (2009) is the perfect starting point for such a model.

\section{References}

Bechara A, Tranel D, Damasio H (2000) Characterization of the decision-making deficit of patients with ventromedial prefrontal cortex lesions. Brain 123:2189-2202.

Damasio A (1994) Descartes' error: emotion, reason, and the human brain. New York: G.P. Putnam's.

Koenigs M, Tranel D (2007) Irrational economic decision-making after ventromedial prefrontal damage: evidence from the Ultimatum Game. J Neurosci 27:951-956.

Koenigs M, Young L, Adolphs R, Tranel D, Cushman F, Hauser M, Damasio A (2007) Damage to the prefrontal cortex increases utilitarian moral judgements. Nature 446:908-911.

Krajbich I, Adolphs R, Tranel D, Denburg NL, Camerer CF (2009) Economic games quantify diminished sense of guilt in patients with damage to the prefrontal cortex. J Neurosci 29:2188-2192.

Moretti L, Dragone D, di Pellegrino G (2009) Reward and social valuation deficits following ventromedial prefrontal damage. J Cogn Neurosci 21:128-140.

Shamay-Tsoory SG, Tibi-Elhanany Y, AharonPeretz J (2007) The green-eyed monster and malicious joy: the neuroanatomical bases of envy and gloating (schadenfreude). Brain 130: 1663-1678. 